\title{
Chronic Myeloid Leukaemia - The Choice of Therapy and Future Perspectives
}

Angelo M Carella, Clara Dellepiane, Davide Lovera, Adalberto Ibatici, Chiara Ghiggi, Andrea Carella and Germana Beltrami

Medical Doctor, Hematologist, Divisione di Ematologia 1, IRCCS Azienda Ospedaliera Universitaria San Martino - IST, Genoa, Italy

\begin{abstract}
Recently, the advent of imatinib has opened a new era in the treatment of chronic myeloid leukaemia (CML), leading to an impressive increase in overall survival rates. Today, many CML patients can expect to survive, if properly managed, likely similar to the general population. Recent progresses in CML stem cell biology have identified new leukogenetic pathways and therapeutic strategies that can be used to target the CML stem cell compartment. These studies have opened new perspectives in CML therapy and have highlighted major strategies for treating, and possibly eradicating, CML in the upcoming years.
\end{abstract}

\section{Keywords}

Chronic myeloid leukaemia, stem cell biology, tyrosine kinase inhibitors, new therapeutic strategies

\begin{abstract}
Disclosure: Angelo M Carella, Clara Dellepiane, Davide Lovera, Adalberto Ibatici, Chiara Ghiggi, Andrea Carella and Germana Beltrami have no conflicts of interest to declare. No funding was received in the publication of this article.

open Access: This article is published under the Creative Commons Attribution Noncommercial License, which permits any non-commercial use, distribution, adaptation and reproduction provided the original author(s) and source are given appropriate credit.

Received: 21 April 2015 Accepted: 12 May 2015 Citation: European Oncology \& Haematology, 2015;11(1):25-9 DOI: 10.17925/EOH.2015.11.01.25

Correspondence: Angelo M Carella, Divisione di Ematologia 1, IRCCS Azienda Ospedaliera Universitaria San Martino - IST, Largo Rosanna Benzi, 10-16132 Genova, Italy.

E: angelomichele.carella@hsanmartino.it.
\end{abstract}

The overwhelming majority of patients diagnosed with chronic phase chronic myeloid leukaemia (CP-CML) can achieve long-term survival with a good quality of life. In a recent review, it has been suggested that CML patients responsive to tyrosine kinase inhibitors (TKIS) might become a chronic disease, such as diabetes or hypertension, requiring maintenance treatment. ${ }^{1}$ If we accept this concept, TKI exposure could raise some problems, such as emerging resistance, chronic toxicity and, last but not least, financial costs. ${ }^{2}$ The initial therapy of $\mathrm{CML}$ is based on three available TKIs. These drugs are able to inhibit the phosphorylative activity of the proteins that are coded by the $B C R-A B L$ fusion gene..$^{3-6}$ The TKIs registered for their activity on BCR-ABL are imatinib, nilotinib and dasatinib. Another compound - bosutinib - has been recently evaluated in first-second line therapy. ${ }^{7}$ A fifth compound - ponatinib has been tested in advanced patients. ${ }^{8}$

Imatinib was introduced in 1998 and changed the therapeutic landscape for patients with CML: it remains the most generally utilised first-line therapy for newly diagnosed $\mathrm{CML}$ patients. A complete haematological response (CHR) is typically achieved by almost all CML patients. The complete cytogenetic remission (CCyR) rate ranges between 60-80\%, the major molecular response (MMR) ranges between 40-60 \% and the complete molecular response (CMR) ranges between 15-30\%. Despite this, about one-third of patients who receive imatinib become resistant or discontinue the drug because of side effects. ${ }^{9-14}$ The progression-free survival (PFS) and overall survival (OS) at 10 years are in the order of 80-85 \%10-13 (see Figure 1). The most important side effects of imatinib are fluid retention, myalgia and fatigue.
Nilotinib is a derivative of imatinib that was designed to target BCR$A B L$ more specifically than imatinib. It is able to inhibit also most mutated forms with the exception of T315I mutation. ${ }^{15}$ When this inhibitor has been used as second-line therapy, the CCyR and MMR were around $50 \%$ and $25 \%$, respectively: ${ }^{16}$ when employed as firstline treatment, the achievement of CCyR and MMR were deeper, faster and superior to imatinib. ${ }^{17-19}$ The major side effects of nilotinib are elevated bilirubin, aspartate and alanine aminotransferase, lipase and amylase and peripheral arterial obliterative disease. More recently, nilotinib has been associated with an increased risk of cardiovascular events, which tend to occur later than ponatinib. ${ }^{19-21}$ These cardiovascular events (ischaemic heart disease, ischaemic cerebrovascular events and peripheral artery disease) occurred in between 6 and $12 \%$ of patients when the drug is delivered at 300 and $400 \mathrm{mg}$ BID doses, respectively.

Dasatinib is an active BCR-ABL, which was designed as a Sarc kinase inhibitor. It is able to inhibit most mutated forms with the exception of the T315I mutation. The results of CCYR and MMR are similar to nilotinib.22 When this inhibitor was employed as first-line treatment, the responses were deeper, faster and superior to imatinib. ${ }^{22}$ The major side effects of this TKI inhibitor are leuco-thrombocytopenia, pleural effusion and, more rarely, pulmonary complications.

The results of bosutinib are generally similar to those of the previously described three TKI inhibitors but the differences in terms of CCYR and MMR with imatinib are less significant.? Diarrhoea and an increase of liver enzymes are the most important side effects. 
Figure 1: Survival with CML over Time - The German CML-Study Group Experience 9/2014

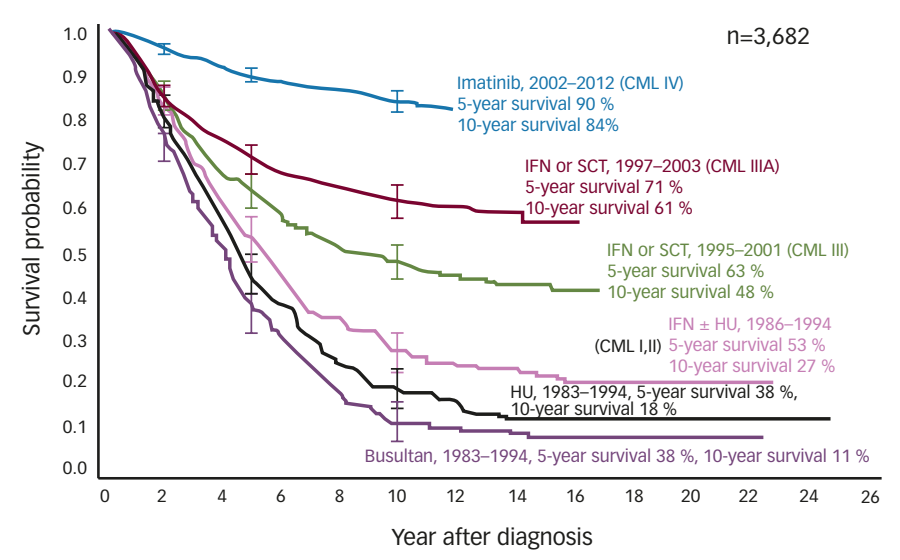

$H U$ = hydroxyurea; IFN = interferon; SCT = stem cell transplantation. Survival with chronic myeloid leukaemia (CML) as observed in five consecutive randomised treatment options studies of the German CML Study Group 1983-2014. Kindly authorised by R Hehlmann.

Ponatinib is a third-generation TKI able to inhibit many mutated forms, including T315I. The safety profile is not yet fully understood but when utilised after first and second-lines TKIs are able to achieve $50 \%$ of response in highly pre-treated patients. ${ }^{23}$ Evidence has recently emerged that ponatinib was associated with a markedly increased risk of arterial occlusion. ${ }^{19,24-26}$ In the Ponatinib Ph+ ALL and CML (PACE) trial, the incidence of arterial occlusion in patients treated with $45 \mathrm{mg}$ daily, was $19 \%$ by 12 months and increased up to $29 \%$ by 24 months. ${ }^{24}$ In order to minimise these risks, the patients should be carefully evaluated for their cardiovascular status prior to ponatinib initiation and setup of adequate prevention measures to reduce the risk.

Other available approaches for the treatment of $\mathrm{CML}$ are: interferon alpha (IFN- $\alpha$ ) and allogeneic transplantation. IFN- $\alpha$ was considered the best drug in the pre-imatinib era when utilised at diagnosis and after autografting..$^{27,28}$ Now it is utilised as pegylated preparations in combination with imatinib in different clinical trials. ${ }^{14}$ The German CMLStudy IV randomised 1,551 CML patients to imatinib $400 \mathrm{mg}$, imatinib plus IFN- $\alpha$, imatinib plus arabinosylcytosine (Ara-C), imatinib after IFN- $\alpha$ failure or imatinib $800 \mathrm{mg}{ }^{29,30}$ The best results were achieved with highdose imatinib and imatinib/IFN- $\alpha$ and these patients obtained molecular remission ${ }^{4,5}$ more quickly than patients on the other study arms. ${ }^{30}$

The French STI571 Prospective Randomized Trial (SPIRIT) assigned more than 600 patients to imatinib $400 \mathrm{mg}$, imatinib $600 \mathrm{mg}$, imatinib plus Ara-C or imatinib plus pegylated (Peg)-IFN- $\alpha .{ }^{31}$ This study demonstrated at 12 , 18 and 24 months significantly faster molecular response rates with imatinib plus PegIFN- $\alpha-2$ a compared with the other study treatments. ${ }^{31}$ Unfortunately, PegIFN- $\alpha$-2a was not well tolerated, so the dose was lowered to $45 \mu \mathrm{g} /$ week. In conclusion, these studies and others confirm that the combination imatinib/FN- $\alpha$ may increase the rate of deep molecular responses but the undesirable side effects of IFN- $\alpha$ can create problems in delivering therapy. Allogeneic stem cell transplantation should not be considered the treatment of choice in the first-line setting. It should be indicated in patients who are in advanced phase of CML or in chronic phase but resistant to first- and second-line TKIs.

\section{Which is the Best First-line Option?}

The best first option is one of the three available TKIs. Both nilotinib and dasatinib are superior to imatinib in terms of CCYR and MMR and the number of patients who progress are lower with these two TKIs than imatinib. The results achieved in CP-CML patients treated with nilotinib showed CCyR achievement at 3 months in about $80 \%$ of them and in more than $90 \%$ at 6 months, whereas the MMR rates where $52 \%$ and $62 \%$, respectively, and more than $80 \%$ at 12 months. ${ }^{17}$ The phase III, randomised multicentre study (Evaluating Nilotinib Efficacy and Safety in clinical Trials [ENEST]), comparing the efficacy and safety of nilotinib versus imatinib in newly diagnosed patients demonstrated that MMR at 1 year was significantly higher for nilotinib (44\%) than for imatinib (22\%) $(p<0.0001)$. Responses were rapidly achieved with nilotinib and were associated with less progression to advanced phases. At the last follow-up, MMR and CMR continued to be higher with nilotinib ${ }^{18,19}$ but the adverse events, in particular cardiovascular, were more frequently associated with it. In another phase III randomised multicentre study, Dasatinib versus Imatinib Study in treatment-naive CML patients (DASISION), the efficacy and safety of dasatinib $100 \mathrm{mg}$ OD was evaluated as the first-line therapy versus imatinib $400 \mathrm{mg} /$ day. ${ }^{32}$ The primary endpoint of the study was CCyR at 1 year; at this time dasatinib achieved more CCyR ( $83 \%$ ) than imatinib $(72 \%)(p<0.001)$; also the MMR rate at 1 year was higher for dasatinib (46\%) than for imatinib (28\%). Moreover, fewer progressions to advanced phase were observed with dasatinib. After 60 months MMR and CMR continued to be higher for dasatinib ( $76 \%$ versus $66 \% ; p=0.002$ ). The adverse events of dasatinib were higher than those of imatinib and were prevalently pleural effusion and myelosuppression; on the contrary, vascular events were not common in the dasatinib group.

Another phase III multicentre study (Bosutinib Efficacy and safety in CNL [BELA]) recently compared bosutinib $500 \mathrm{mg}$ OD versus imatinib $400 \mathrm{mg}$ OD. ${ }^{33}$ The CCyR at 1 year was the primary endpoint of this study but no significant difference was found between the two drugs. It is possible that the results of bosutinib were penalised by drugrelated events and discontinuation of the bosutinib arm. Despite this, molecular remission was higher for bosutinib (39\%) than for imatinib (26\%) $(p=0.002)$.

More recently, great importance was given to a faster time to achieve CCyR and, most importantly, BCR-ABL transcript reduction $<10 \%$ at 3 months. ${ }^{34-45}$ All authors demonstrated that an early molecular response is able to predict the improvement of overall survival.

\section{What is our Opinion on the Best TKI Approach as Front-line Therapy?}

Imatinib has changed the landscape of CML. The cost of the drug is not low and this has certainly reduced the use of this drug in many lowincome countries. In the near future, hopefully imatinib will be available as a generic (as happened already in Turkey) and this could be save more patients. On the other hand, two other TKIs, dasatinib and nilotinib, are potential alternatives to imatinib as first-line therapy for $\mathrm{CML}$, mainly because of the deeper and faster molecular responses induced by these drugs. ${ }^{18,32}$ In our opinion, imatinib should be used in low-risk patients while the second-generation TKIs should be offered to intermediate- and highrisk patients only. An alternative should be to initially start with imatinib $400 \mathrm{mg}$ and then to switch to a second-generation TKIs as soon as a nonoptimal response has been achieved. Of course, comorbidities and sideeffects of TKIs should be considered in the final decision. Finally, if we can evaluate the mutations at diagnosis in high-risk patients, we could be able to find mutations that could be sensible to imatinib or other TKIs; in this way, we can offer the most sensible TKI for that specific mutation. Our recent retrospective experience permitted us to find mutations at diagnosis mainly in high-but not in low-risk patients. ${ }^{46}$ 


\section{The Basis of Treatment for Tomorrow}

Recent progress in our understanding of CML biology has highlighted new oncogenic pathways and resistance mechanisms that are considered as highly promising targets for achieving better control of this disease and, possibly, its cure. In this context, work from Tessa Holyoake's lab in Glasgow showed that the majority of CML progenitor (CD34+) cells undergo division in culture in the presence of growth factors. ${ }^{47}$ When TKIs were added to the culture, the proliferating cells were killed, while dormant cells that do not divide were completely refractory to the drugs. ${ }^{48}$ These dormant or quiescent cells are probably responsible for the 'molecular persistence of the disease', namely, the residual low level of BCR-ABL transcript positivity that is detected by quantitative polymerase chain reaction (PCR) in many cases. since the available TKIs seem not to be able to eliminate dormant cells, the question is how to devise alternative biological strategies to eradicate these cells. Recent studies from different groups have provided encouraging potential therapeutic approaches, recently discussed in two recent CML workshops at the American Society of Hematology (ASH). ${ }^{49,50}$ As discussed by Perrotti et al., protein phosphatase 2A (PP2A) is a tumour suppressor whose activity is inhibited in Philadelphia (Ph)-positive leukaemias, but not in normal haematopoietic stem/progenitor cells. . $^{51,52}$ Fingolimod (FTY720), an immunosuppressive synthetic sphingosine analogue, ${ }^{53}$ has the capacity to re-activate PP2A and shows limited toxicity on normal cells. FTY720 indeed has anti-leukaemic activity on CD34+ cells from TKI-sensitive and TKI-resistant CML progenitors. ${ }^{54-57}$ Notably, in primitive CML cells, the pro-apoptotic effect of FTY720 might not require BCR-ABL activity, ${ }^{53}$ which, as reported, is dispensable for their survival. ${ }^{58}$ Since FTY720 is active on oncogene-driven pathways other than the one controlled by BCR-ABL and preserves normal cells at the same time, it is not surprising that this is also effective in other types of leukaemia.

Another interesting approach for treating CML is the inhibition of autophagy. CML cells that survive TKI treatment show features that are reminiscent of those observed in response to growth factor deprivation, such as a reduced size and a marked increase in cytoplasmic vacuoles. ${ }^{59}$ These cellular adaptations reflect the activation of autophagy, a cellrecycling process that relies on a specific self-digestion apparatus and confers the ability to survive stress conditions and inhospitable environments. ${ }^{60} \mathrm{~A}$ classic marker of autophagy-activation is represented by the cleavage and lipid modification of the Atg protein LC3, which, as a result of these modifications, is able to bind to the internal membrane of autophagic vacuoles and to act as a docking site for proteins to be 'digested'. Treatment of CML stem cells with TKIs induces LC3 conversion and activates the autophagic programme, thus contributing to the CML stem cell resistance of these agents. ${ }^{61}$ These observations provided the rationale for combining TKIs, which kill only mature BCR-ABL+ cells, with autophagy inhibitors, such as chloroquine or hydroxychloroquine, to eradicate the $\mathrm{CML}$ stem cell compartment. ${ }^{62} \mathrm{~A}$ clinical trial based on these interesting results is ongoing in the UK; patients who achieve a MMR after 1 year of imatinib treatment are randomised to continue imatinib alone or imatinib plus chloroquine. Follow-up data are not available yet.

Recent laboratory studies with macrolides, such as clarithromycin, have demonstrated that these antibiotics enhance the activity of TKIs in CML cells. The mechanism of induction of cell death by clarithromycin combined with TKIs appears to rely on the inhibition of the late stage of autophagy. This is clearly demonstrated by an increase in LC3-II protein levels and by a concomitant increase in cellular vacuole formation. ${ }^{63}$ Remarkable responses obtained in seven patients with advanced
CML who were treated in Genoa with clarithromycin plus TKIs indeed support the hypothesis that autophagy inhibition may make CML cells sensitive to killing by TKIs. ${ }^{64,65}$

The oncogenic Hedgehog (sHH) signalling plays a crucial role in cancer because of its involvement in chromatin remodelling, cell cycle control and apoptosis. ${ }^{66}$ The expression of the sHH effectors Gli-1 and, in particular, Gli-2, is significantly increased in both chronic and accelerated phase CML progenitors. ${ }^{67,68}$

Finally, drugs with the ability to block BCR-ABL mutated forms that are otherwise resistant to traditional TKIs are also a new frontier in the $C M L$ therapeutics arena, and some of these agents have already reached advanced clinical evaluation phases. The T315I BCR-ABL-mutant protein is resistant to imatinib, dasatinib, nilotinib and bosutinib, and remains a major concern for clinicians. Among the new TKIs, ponatinib is active against this type of mutated BCR-ABL. Ponatinib, in particular, is considered a pan-BCR-ABL inhibitor, which potently inhibits the T315I BCR-ABL mutant and overcomes mutation-based resistance. ${ }^{69,70}$

\section{Discontinuation of TKI Therapy - Is it Possible?}

Long-term TKI treatment leads to a gradual reduction of residual disease in the majority of patients and in some patients no residual disease is detectable with real-time quantitative (RQ)-PCR. The question now is: can TKI be discontinued in these particular group of patients, since such a possibility would have a major impact on patient quality of life and, in terms of costs, on healthcare systems? Recently a variety of clinical studies have evaluated the possibility of exploring the TKI discontinuation in patients with stable molecular remission. After the first positive results achieved with imatinib discontinuation, ${ }^{71}$ a pilot multicentre study entitled Stop imatinib (STIM) was organised; 100 patients with $\mathrm{CP}-\mathrm{CML}$ and undetectable BCR-ABL transcript for at least 2 years after imatinib were followed. After 1 year, $41 \%$ of patients were molecular relapse-free survivors. ${ }^{72} \mathrm{~A}$ recent update at 5 years confirmed a cumulative incidence of $61 \%$ of molecular relapses with three patients relapsed at 19, 20 and 22 months. ${ }^{73}$ All molecular relapsed patients were responsive to re-treatment with imatinib. Similar results were achieved with Australia ${ }^{74}$ and Japan ${ }^{75}$ teams. In the attempt to define prognostic markers to increase the rate of patients in molecular response after TKIs discontinuation, the preliminary results of the European Stop Tyrosine Kinase Inhibitor (EURO-SKI) study have been presented at the last ASH meeting. Seven hundred patients entered the trial. An interim analysis of the first 200 patients from eight European countries with a minimum follow-up of 12 months was presented. One hundred and eleven (56\%) of the 200 patients remained therapy-free after the discontinuation of TKIs and 89 relapsed at a molecular level. Similar to the STIM study, most relapses occurred quickly within the first 6 months. The duration of prior TKI treatment and the achievement of MR4 for more than 5 years before discontinuation seemed to have a positive effect. Moreover, the estimated drug-related savings to healthcare systems in the eight EURO-SKI countries was estimated to be €7 million.

The STOP-2G-TKI study, conducted by the French CML study group, recruited 52 patients. ${ }^{76}$ The inclusion criteria of this study required prior second-generation TKI treatment with achievement of MR4.5 remission for at least 2 years. Fifty-two patients with a median of 6.5 years of TKI treatment and a median duration of MR4.5 of 2.3 years, have been evaluated and followed for 12-60 months. Twenty-four (46\%) of the 52 patients experienced molecular relapse and had to restart 
treatment. One relapse occurred 24 months and one 37 months after TKI discontinuation.

In conclusion, according to these results, it seems obvious that patients need to be regularly monitored even years after stopping treatment. The good news is that the molecular relapsed patient, after restarting treatment, achieved a deep molecular response and, until now, none of the patients progressed to advanced CML phases. It may be concluded that molecular response and imatinib treatment were the most important predictors for successful therapy. Recently, of great interest, are two abstracts presented at the last ASH meeting. The first demonstrated that age $<45$ years combined to detection of BCR-ABL in a more sensitive 'digital $\mathrm{PCR}^{\prime}$ test were associated with an increasing risk of relapse. ${ }^{77}$ The second abstract (Korean Imatinib Discontinuation Study [KIDS]) confirmed that both duration of imatinib treatment and of deep molecular response were the most important predictors for successful discontinuation. ${ }^{78}$

\section{Conclusions}

Recent progress in CML stem cell biology has identified several main molecules and signalling pathways that are involved in the maintenance of CML stem cells, offering possibilities for exploring new therapies for this disease. However, much work remains to be carried out - work that should benefit new CML patients in the future and, hopefully, also patients with other forms of cancer whose management may be based on principles derived from pioneering work in CML.
Hehlmann R, CML - where do we stand in 2015? Ann Hematol, 2015;94(Suppl. 2):S105-257.

2. Melo JV, Ross DM, Minimal residual disease and discontinuation of therapy in chronic myeloid leukemia: can we aim at a cure?, Hematology Am Soc Hematol Educ Program, 2011;136-42.

3. Hehlmann R, Hochhaus A, Baccarani M, European Leukemianet. Chronic myeloid leukaemia, Lancet, 2007:370(9584):342-50.

4. Quintás-Cardama A, Cortes J, Molecular biology of bcr-abl1positive chronic myeloid leukemia, Blood, 2009;113:1619-30

5ardiman JW, Melo JV, Baccarani M, et al., Chronic myelogenous leukemia, BCR-ABL1 positive. In: Swerdlow SH, Campo E, Harris NL, et al., Eds, WHO Classification of Tumours of Hematopoietic and Lymphoid Tissues, Lyon, France: IARC, 2008;32-7

6. Baccarani M, Cortes J, Pane F, et al., European LeukemiaNet. Chronic myeloid leukemia: an update of concepts and management recommendations of European Leukemianet, J Clin Oncol, 2009;27:6041-51.

7. Cortes JE, Maru A, De Souza CA, et al., Bosutinib versus Imatinib in newly diagnosed chronich phase chronic myeloid leukemia - BELA trial: 24-month follow-up, Blood, 2011:118:455.

8. Cortes JE, Kim DW, Pinilla-Ibarz J, et al., PACE Investigators. A phase 2 trial of ponatinib in Philadelphia chromosome-
positive leukemias, N Eng/ J Med, 2013;369:1783-96.

O'Brien SG, Guilhot F, Larson RA, et al., IRIS Investigators Imatinib compared with interferon and low-dose cytarabine for newly diagnosed chronic-phase chronic myeloid leukemia, N Eng/ J Med, 2003;348:994-1004.

10. Hughes TP, Kaeda J, Branford S, et al., International Randomised Study of Interferon versus STI571 (IRIS) Study Group. Frequency of major molecular responses to imatinib or interferon alfa plus cytarabine in newly diagnosed chronic myeloid leukemia, N Eng/ J Med, 2003;349:1423-32.

11. Druker BJ, Guilhot F, O'Brien SG, et al., IRIS Investigators Five-year follow-up of patients receiving imatinib for chronic Five-year follow-up of patients receiving imatinib 17.

12. Hochhaus $A$, O'Brien $S G$, Guilhot $F$, et al., IRIS Investigators Six-year follow-up of patients receiving imatinib for the first-line treatment of chronic myeloid leukemia, Leukemia 2009;23:1054-61.

13. Baccarani M, Rosti G, Castagnetti F, et al., Comparison of imatinib $400 \mathrm{mg}$ and $800 \mathrm{mg}$ daily in the front-line treatment of high-risk, Philadelphia-positive chronic myeloid leukemia: European LeukemiaNet Study, Blood, 2009;113:4497-504.

14. HehImann R, Lauseker M, Jung-Munkwitz S, et al., Tolerabilityadapted imatinib $800 \mathrm{mg} / \mathrm{d}$ versus $400 \mathrm{mg} / \mathrm{d}$ versus 400 $\mathrm{mg} / \mathrm{d}$ plus interferon- $\alpha$ in newly diagnosed chronic myeloid leukemia, J Clin Oncol, 2011;29:1634-42

15. Soverini S, Hochhaus A, Nicolini FE, et al., BCR-ABL kinase domain mutation analysis in chronic myeloid leukemia patients treated with tyrosine kinase inhibitors: recommendations from an expert panel on behalf of recommendations from an expert panel on behalf

16. Kantarjian HM, Giles FJ, Bhalla KN, et al., Nilotinib is effective in patients with chronic myeloid leukemia in chronic phase after imatinib resistance or intolerance: 24 -month follow-up results. Blood, 2011;117:1141-5

17. Rosti G, Palandri F, Castagnetti F, et al., GIMEMA CML Working Party. Nilotinib for the frontline treatment of $\mathrm{Ph}(+)$ chronic myeloid leukemia, Blood, 2009;114:4933-8.

18. Saglio G, Kim DW, Issaragrisil S, et al., ENESTnd Investigators Nilotinib versus imatinib for newly diagnosed chronic myeloid leukemia, N Eng/ J Med, 2010;362:2251-9.

19. Saglio G, Hughes TB, Clark RE, et al., ENESTnd update: Nilotinib vs imatinib in patients with newly diagnosed CML in chronic phase and the impact of early molecular response and Sokal risk at diagnosis on long-term outcomes, Blood, 2013;122:92.

20. Aichberger KJ, Herndlhofer S, Schernthaner GH, et al Progressive peripheral arterial occlusive disease and other vascular events during Nilotinib therapy in CML, Am 」 Hematol, 2011:86:533-9.

21. Le Coutre P, Rea D, Abruzzese E, et al., Severe peripheral arterial disease during nilotinib therapy, J Natl Cancer Inst,
2011;103:1347-8.

22. Hochhaus A, Kantarjian HM, Baccarani M, et al., Dasatinib induces notable hematologic and cytogenetic responses in chronic-phase chronic myeloid leukemia after failure of imatinib therapy, Blood, 2007, 109:2303-9.

23. Cortes JE, Kantarjian $\mathrm{H}$, Shah NP, et al., Ponatinib in refractory Philadelphia chromosome-positive leukemias, N Eng/ J Med, 2012:367:2075-2088.

24. Cortes JE, Kim D-W, Pinilla-lbarz J, et al., Ponatinib in patients (pts) with chronic myeloid leukemia (CML) and Philadelphia chromosome-positive acute lymphoblastic leukemia ( $\mathrm{Ph}+$ ALL) resistant or intolerant to dasatinib or nilotinib, or with the T315I BCR-ABL mutation: 2-year follow-up of the PACE trial, Blood, 2013;122:abstract 650 .

25. Cortes JE, Hochhaus A, Kim D-W, et al., Four-year (yr) followup of patients (pts) with newly diagnosed chronic myeloid leukemia in chronic phase (CML-CP) receiving dasatinib or imatinib: efficacy based on early response, Blood, 2013;122:Abstract 653

26. Cortes JE, Kantarjian HM, Khoury HJ, et al., Long-term evaluation of vascular toxicity in patients with $\mathrm{Ph}+$ leukemias treated with bosutinib, I Clin Oncol, 2014;32 (Suppl. 15):Abstract 7060.

27. Carella AM Lerma E, Corsetti MT, et al ., Autografting with Philadelphia chromosome-negative mobilized hematopoieitic progenitor cells in chronic myelogenous leukemia, Blood, 1999;93:1534-9.

28. Carella AM, Cunningham I, Lerma E, et al., Mobilization and transplantation of Philadelphia-negative peripheral-blood progenitor cells early in chronic myelogenous leukemia, J Clin Oncol, 1997;15:1575-82.

29. Hehlmann R, Lauseker M, Jung-Munkwitz S, et al., Tolerabilityadapted imatinib $800 \mathrm{mg} / \mathrm{d}$ versus $400 \mathrm{mg} / \mathrm{d}$ versus $400 \mathrm{mg} / \mathrm{d}$ plus interferon-alpha in newly diagnosed chronic myeloid leukemia, J Clin Oncol, 2011;29:1634-42.

30. HehImann R, Muller MC, Lauseker M, et al., Deep molecular response is reached by the majority of patients treated with imatinib, predicts survival, and is achieved more quickly by optimized high-dose imatinib: results from the randomized CML-study IV, I Clin Oncol, 2014:32:415-23.

31. Preudhomme C, Guilhot J, Nicolini FE, et al., Imatinib plus peginterferon alfa-2a in chronic myeloid leukemia, N Eng/ J Med, 2010;363:2511-21.

32. Kantarjian $\mathrm{H}$, Shah NP, Hochhaus A, et al., Dasatinib versus matinib in newly diagnosed chronic-phase chronic myeloid leukemia, N Engl J Med, 2010;362:2260-70.

33. Cortes JE, Kim DW, Kantarijan HM, et al., Bosutinib vs imatinib in newly diagnosed chronic phase CML: results from the BELA trial, J Clin Oncol, 2012;30:3486-92.

34. Kantarijan H, Hochhaus A, Saglio G, et al., Nilotinib versus imatinib for the treatment of patients with newly diagnosed chronic phase, Philadelphia chromosome-positive, chronic myeloid leukaemia: 24-month minimum follow-up of the phase 3 randomised ENESTnd trial, Lancet Oncol, 2011:12:841-51.

35. Kantariian HM, Shah NP, Cortes JE, et al., Dasatinib or imatinib in newly diagnosed chronic-phase chronic myeloid leukemia: 2-year follow-up from a randomized phase 3 trial (DASISION) Blood, 2012;119:1123-9.

36. Wang L, Pearson K, Ferguson JE, et al., The early molecular response to imatinib predicts cytogenetic and clinica outcome in chronic myeloid leukaemia, Br $\mathrm{J}$ Haematol 2003;120:990-9.

37. Branford S, Rudzki Z, Harper A, et al., Imatinib produces significantly superior molecular responses compared to interferon alfa plus cytarabine in patients with newly diagnosed chronic myeloid leukemia in chronic phase, Leukemia, 2003;17:2401-9.

38. Hughes TP, Hochhaus A, Branford S, et al., IRIS investigators. Long-term prognostic significance of early molecular response to imatinib in newly diagnosed chronic myeloid leukemia: an analysis from the International Randomized Study of Interferon and STI571 (IRIS), Blood, 2010;116:3758-65.

39. Marin D, Hedgley C, Clark RE, et al., Predictive value of early molecular response in patients with chronic myeloid leukemia treated with first-line dasatinib, Blood, 2012;120:291-4.
40. Marin D, Ibrahim AR, Lucas C, et al., Assessment of BCRABL1 transcript levels at 3 months is the only requirement for predicting outcome for patients with chronic myeloid leukemia treated with tyrosine kinase inhibitors, I Clin Oncol, 2012;30:232-8.

41. Hanfstein B, Müller MC, Hehlmann R, et al., German CML Study Group. Early molecular and cytogenetic response is predictive for long-term progression-free and overall survival in chronic myeloid leukemia (CML), Leukemia, 2012;26:2096-102.

42 Branford S, Kim DW, Soverini S, et a Initial molecular response at 3 months may predict both response and eventfree survival at 24 months in imatinib-resistant or -intolerant patients with Philadelphia chromosome-positive chronic myeloid leukemia in chronic phase treated with nilotinib J Clin Oncol, 2012;30:4323-9.

43. Jain P, Kantarjian $\mathrm{H}$, Nazha A, et al., Early responses predict better outcomes in patients with newly diagnosed chronic myeloid leukemia: results with four tyrosine kinase inhibitor modalities, Blood, 2013:121:4867-74.

44. Hughes TP, Lipton JH, Spector N, et al., Deep molecula responses achieved in patients with $\mathrm{CML}-\mathrm{CP}$ who are switched to nilotinib after long-term imatinib, Blood, 2014:124:729-36.

45. Marin $\mathrm{D}$, Initial choice of therapy among plenty for newly diagnosed chronic myeloid leukemia, Hematology Am SOC Hematol Educ Program, 2012;2012:115-21.

46. Carella AM, Garuti A, Cirmena G, et al., Kinase domain mutations of BCR-ABL identified at diagnosis before imatinibbased therapy are associated with progression in patients with high Sokal risk chronic phase chronic myeloid leukemia, Leukemia \& Lymphoma, 2010;51:275-8.

47. Graham SM, Jørgensen HG, Allan E, et al., Primitive, quiescent, Philadelphia-positive stem cells from patients with chronic myeloid leukemia are insensitive to STI571 in vitro, Blood, 2002;99:319-25

48. Copland M, Hamilton A, Elrick $L$, et al., Dasatinib (BMS354825) targets an earlier progenitor population than imatinib in primary CML but does not eliminate the quiescent fraction. Blood, 2006:107:4532-9.

49. Carella AM, Goldman JM, Martinelli G, et al., Chronic myeloid leukemia: the basis of treatment for tomorrow, Haematologica, 2011;96:1737-9.

50. Carella AM, Branford S, Deininger M, et al., What challenges remain in chronic myeloid leukemia research? Haematologica, 2013;98:1168-72.

51. Neviani P, Santhanam R, Trotta R, et al., The tumor suppressor PP2A is functionally inactivated in blast crisis CML through the inhibitory activity of the BCR/ABL-regulated SET protein, Cancer Cell, 2005:8:355-68.

52. Walker $\mathrm{C}$, Oaks JJ, Neviani P, et al., PP2A Activating Drugs (PAD): Anti-leukemic and non-toxic activity of two novel and non-immunosuppressive FTY720 derivatives, Blood, 2010:116:2901.

53. Aktas O, Kury P, Kieseier B, et al., Fingolimod is a potential novel therapy for multiple sclerosis, Nat Rev Neurol, 2010:6:373-82.

54. Neviani P, Santhanam R, Oaks JJ, et al., FTY720, a new alternative for treating blast crisis chronic myelogenous leukemia and Philadelphia chromosome-positive acute Iymphocytic leukemia, I Clin Invest, 2007;117:2408-21.

5. Neviani P, Harb JG, Oaks JJ, et al., BCR-ABL1 kinase activity but not its expression is dispensable for $\mathrm{Ph}+$ quiescent stem cell survival which depends on the PP2A-controlled Jak2 activation and is sensitive to FTY720 treatment, Blood, 2010;116:515

56. Oaks JJ, Mukhopadhyay A, Santhanam R, et al., Pharmacologic restoration of PP2A activity and interference with the SETPP2A interplay by FTY720 and its non-immunosuppressive derivative as a novel and efficient therapy for Ph-negative myeloproliferative disorders, Blood, 2010;116:775.

57. Roberts KG, Smith AM, MCDougall F, et al., Essentia requirement for PP2A inhibition by the oncogenic receptor $\mathrm{C}-\mathrm{KIT}$ suggests PP2A reactivation as a strategy to treat C-KIT+ cancers, Cancer Res, 2010;70:5438-47.

58. Corbin AS, Agarwal A, Loriaux M, et al., Human chronic myeloid leukemia stem cells are insensitive to imatinib despite inhibition of BCR-ABL activity, I Clin Invest, 
2011;121:396-409.

59. Bellodi C, Lidonnici MR, Hamilton A, et al., Targeting autophagy potentiates tyrosine kinase inhibitor-induced cell death in Philadelphia chromosome-positive cells, including primary CML stem cells, J Clin Invest, 2009;119:1109-23.

60. Kroemer G, Mariño G, Levine B, Autophagy and the integrated stress response, $\mathrm{Mol}$ Cell, 2010;40:280-93.

61. Ertmer $A$, Huber $V$, Gilch $S$, et al., The anticancer drug imatinib induces cellular autophagy, Leukemia, 2007;21:936-42.

62. Helgason GV, Karvela M, Holyoake TL, Kill one bird with two stones: potential efficacy of BCR-ABL and autophagy inhibition in CML, Blood, 2011;118:2035-43.

63. Altman JK, Platanias LC, A new purpose for an old drug: inhibiting autophagy with clarithromycin, Leuk Lymphoma, 2012;53:1255-6.

64. Carella AM, Beltrami G, Pica G, et al., Clarithromycin potentiates tyrosine kinase inhibitor treatment in patients with resistant chronic myeloid leukemia, Leuk Lymphoma 2012;53:1409-11.

65. Schafranek L, Leclercq TM, White DL, et al., Clarithromycin Enhances Dasatinib-Induced Cell Death in CML cells, by inhibition of late stage autophagy, Leuk Lymphoma, 2013;54:198-201.

66. Zhao C, Chen $\mathrm{A}$, Jamieson $\mathrm{CH}$, et al., Hedgehog signalling is essential for maintenance of cancer stem cells in myeloid leukaemia, Nature, 2009;458:776-9.

67. Dierks C, Beigi R, Guo G.R, et al., Expansion of Bcr-Abl-positive leukemic stem cells is dependent on Hedgehog pathway activation, Cancer Cell, 2008;14:238-49.

68. Irvine DA, Heaney NB, Holyoake TL, Optimising chronic myeloid leukaemia therapy in the face of resistance to tyrosine kinase inhibitors - A synthesis of clinical and laboratory data, Blood Rev, 2010;24:1-9.

69. O'Hare T, Shakespeare W. Chu X et al, AP24534, a pan-BCRABL inhibitor for chronic myeloid leukemia, potently inhibits ABL inhibitor for chronic myeloid leukemia, potently inhibits
the T315I mutant and overcomes mutation-based resistance the T315I mutant and overcome

70. Zhou T, Commodore L, Huang WS, et al., Structura mechanism of the Pan-BCR-ABL inhibitor ponatinib (AP24534): lessons for overcoming kinase inhibitor resistance, Chem Biol Drug Des, 2011;77:1-11.

71. Rousselot $P$, Huguet $F$, Rea $D$, et al., Imatinib mesylate discontinuation in patients with chronic myelogenous leukemia in complete molecular remission for more than 2 years, Blood, 2007; 109:58-60.

72. Mahon FX, Réa D, Guilhot J, et al., Intergroupe Français des Leucémies Myéloïdes Chroniques. Discontinuation of have maintained complete molecular remission for at least 2 years: the prospective, multicentre Stop Imatinib (STIM) trial, Lancet Oncol, 2010;11:1029-35.

73. Mahon FX, Long term follow-up after imatinib cessation for patients in deep molecular response: The update results of the STIM1 study, Proc ASH, 2013;Abstract 225.

74. Ross DM, Branford S, Seymour JF, et al., Safety and efficacy of imatinib cessation for CML patients with stable undetectable minimal residual disease: results from the TWISTER study Blood, 2013;122:515-22.

75. Takahashi N Kyo T, Maeda Y, et al., Discontinuation of imatinib in Japanese patients with chronic myeloid leukemia, Haematologica, 2012;97:903-6.

76. Rea D, Rousselot P, Nicolini F et al., Discontinuation of dasatinib and nilotinib in CML patients with stable undetectable BCR-ABL transcripts: results from the French CML Group(FI-LMC), Blood (ASH Annual Meeting Abstracts), 2012;120:Abstract 916.

77. Mori S, Vagge E, Le Coutre PH, et al., The risk of relapse in CML patients who discontinued imatinib can be predicted based on patients age and the results of dPCR analysis, Blood, 2014:15:Abstract 632.

78. Yun Jeon OH, Soo Young Choi, Sung Eun Lee, et al. Results from Korean Imatinib Discontinuation Study (KIDs): updated data with 14-month median follow-up, Blood, 2013;15:122. 